\title{
Direct and compound interactions for the neutron-induced fission cross section determination
}

\author{
V.M. Maslov \\ Joint Institute for Nuclear and Energy Research, 220109 Minsk-Sosny, Belarus
}

\begin{abstract}
Recent ratio surrogate data for the neutron-induced fission cross sections are inter-compared for the chain of Th and $\mathrm{U}$ nuclides. The structure of the fission barrier and the angular moments of the entrance channel states are shown to be significant. The excitation/position of the $\mathrm{K}^{\pi}=2^{+}$abnormal rotational band-head at the inner saddle of even-even $U$ fissioning nuclide like ${ }^{240} \mathrm{U}$ is shown to be critically important. At ${ }^{231} \mathrm{Th}$ nuclide medium excitations of $10-20 \mathrm{MeV}$, when pre-fission neutron emission is possible in the reaction ${ }^{232} \mathrm{Th}\left({ }^{3} \mathrm{He},{ }^{4} \mathrm{He}\right)$, the excitation of $\mathrm{K}^{\pi}=0^{-}$states depends on the massasymmetry of the even nuclide ${ }^{230} \mathrm{Th}$ saddle shapes. Octupole band states are shown to be relevant for the ${ }^{232} \mathrm{Th}\left({ }^{3} \mathrm{He},{ }^{3} \mathrm{He}^{\prime}\right)$ reaction. The consistency of surrogate, ratio surrogate and neutron-induced fission data is revisited.
\end{abstract}

\section{Introduction}

Pick-up, stripping or inelastic scattering reactions of charged particles beams with stable actinides targets are used nowadays for the determination of neutron-induced fission cross section of short-lived target nuclides. The first positive experience in a wide excitation energy range was for the inelastic scattering reactions, followed by fission: ${ }^{238} \mathrm{U}\left(\alpha, \alpha^{\prime} \mathrm{F}\right)$ and ${ }^{236} \mathrm{U}\left(\alpha, \alpha^{\prime} \mathrm{F}\right)$ by Burke et al. [1]. These ratio surrogate fission data, which are generally consistent with surrogate data by Britt et al. [3], renormalized by Younes and Britt [4,5], supported the ${ }^{237} \mathrm{U}(\mathrm{n}, \mathrm{F})$ cross section, predicted by Maslov [2] for incident neutron energies up to $20 \mathrm{MeV}$. The prediction was based on the consistent description of ${ }^{238} \mathrm{U}(\mathrm{n}, \mathrm{F}),{ }^{238} \mathrm{U}(\mathrm{n}, \mathrm{xn})$ cross sections and ${ }^{238} \mathrm{U}(\mathrm{n}, \mathrm{F})$ prompt fission neutron spectra in the emissive fission domain [6].

The stripping reactions data ${ }^{238} \mathrm{U}\left({ }^{18} \mathrm{O},{ }^{16} \mathrm{OF}\right)$ and ${ }^{234} \mathrm{U}\left({ }^{18} \mathrm{O},{ }^{16} \mathrm{OF}\right)$ by Burke et al. [7], which may simulate the ratio of ${ }^{239} \mathrm{U}(\mathrm{n}, \mathrm{F})$ and ${ }^{235} \mathrm{U}(\mathrm{n}, \mathrm{F})$ reaction cross sections, turned out to be discrepant with the stripping reaction ${ }^{238} \mathrm{U}\left({ }^{3} \mathrm{H}, \mathrm{pf}\right)$ data [5] in the first-chance fission domain. These inconsistencies of ratio surrogate [7] and surrogate data [3-5], could stem from the angular momentum differences of the entrance channel. These differences may cancel out, like in case of $\left(\alpha, \alpha^{\prime} \mathrm{F}\right)$, but may be "amplified", when stripping reaction $\left({ }^{18} \mathrm{O},{ }^{16} \mathrm{O}\right)$ is used. Another factor, which might be important, is the structure of the fission barrier, on which the transition states evidences are dependent. In case of ${ }^{238,240} \mathrm{U}$ fissioning nuclides pronunciation of the transition states ordering at inner saddle is probed.

The surrogate data for the ${ }^{230} \mathrm{Th}(\mathrm{n}, \mathrm{F})$ reaction are inferred from the ratio of fission probabilities of ${ }^{231} \mathrm{Th}$ and ${ }^{235} \mathrm{U}$ nuclides by Goldblum et al. [8]. The desired compound nuclei were populated using a pick-up reactions ${ }^{232} \mathrm{Th}\left({ }^{3} \mathrm{He},{ }^{4} \mathrm{He}\right)$ and ${ }^{236} \mathrm{U}\left({ }^{3} \mathrm{He},{ }^{4} \mathrm{He}\right)$. At medium excitations of $10-15 \mathrm{MeV}$, when pre-fission neutron emission is possible, the excitation of $\mathrm{K}^{\pi}=0^{-}$states depends on the massasymmetry of the even-even nuclide ${ }^{230} \mathrm{Th}$ saddle shapes.

The surrogate data for the ${ }^{231} \mathrm{Th}(\mathrm{n}, \mathrm{F})$ reaction are inferred from the ratio of fission probabilities of ${ }^{232} \mathrm{Th}$ and ${ }^{236} \mathrm{U}$ nuclides by Goldblum et al. [8]. The desired compound nuclei are populated using

\footnotetext{
a e-mail: maslov@sosny.bas-net.by
}

This is an Open Access article distributed under the terms of the Creative Commons Attribution-Noncommercial License 3.0, which permits unrestricted use, distribution, and reproduction in any noncommercial medium, provided the original work is properly cited. 
inelastic scattering reactions ${ }^{232} \mathrm{Th}\left({ }^{3} \mathrm{He},{ }^{3} \mathrm{He}^{\prime}\right)$ and ${ }^{236} \mathrm{U}\left({ }^{3} \mathrm{He},{ }^{3} \mathrm{He}^{\prime}\right)$ by Goldblum et al. [8]. Even nuclide ${ }^{232}$ Th mass-asymmetric saddle shape is probed in that case. Of importance here is also the excitation of $\mathrm{K}^{\pi}=0^{-}$states, which depends on the mass-asymmetry of the even-even nuclide ${ }^{232} \mathrm{Th}$ saddle shapes.

The statistical theory approach, tuned via consistent description of ${ }^{232} \mathrm{Th}(\mathrm{n}, \mathrm{F}),{ }^{232} \mathrm{Th}(\mathrm{n}, 2 \mathrm{n})$ and prompt fission neutron spectra in the emissive fission domain, allowed to estimate ${ }^{230} \mathrm{Th}(\mathrm{n}, \mathrm{F})$, ${ }^{231} \mathrm{Th}(\mathrm{n}, \mathrm{F})$ and ${ }^{229} \mathrm{Th}(\mathrm{n}, \mathrm{F})$ [9]. Estimated in [9] ${ }^{230} \mathrm{Th}(\mathrm{n}, \mathrm{F})$ fission cross section is confirmed by the ratio surrogate data of [8]. However, the ratio surrogate data of ${ }^{231} \mathrm{Th}(\mathrm{n}, \mathrm{F})$ [8] appear to be drastically discrepant with the fission probability of ${ }^{232} \mathrm{Th}$, extracted from ${ }^{232} \mathrm{Th}(\mathrm{n}, \mathrm{F})$ [8] and stripping reaction ${ }^{230} \mathrm{Th}(\mathrm{t}, \mathrm{pf})$ fission probability data [3-5].

The set of surrogate, ratio surrogate data for ${ }^{230,231} \mathrm{Th}(\mathrm{n}, \mathrm{F})$ and ${ }^{237,239} \mathrm{U}(\mathrm{n}, \mathrm{F})$ reactions allows to consider their consistency with the data of neutron interactions with respective target nuclides. They support the predicted sharing of the (n, F) cross sections into (n, xnf) contributions, specifically, the increasing trend of the first-chance fission cross section for ${ }^{230} \mathrm{Th}$ and ${ }^{237,239} \mathrm{U}$ targets, posing a problem for the ${ }^{231} \mathrm{Th}(\mathrm{n}, \mathrm{F})$ fission cross section.

\section{$2{ }^{237} U(n, F)$ and ${ }^{239} U(n, F)$ reactions}

The neutron induced fission cross section of ${ }^{237} \mathrm{U}(\mathrm{n}, \mathrm{F})$ is essentially defined by the ${ }^{238} \mathrm{U}$ fission and level density parameters. They could be extracted fixing the contribution of the ${ }^{238} \mathrm{U}(\mathrm{n}, \mathrm{nf})$ reaction to the observed fission cross section of ${ }^{238} \mathrm{U}(\mathrm{n}, \mathrm{F})$. Above emissive fission threshold contributions to the observed ${ }^{238} \mathrm{U}(\mathrm{n}, \mathrm{F})$ fission cross section coming from $(\mathrm{n}, \mathrm{xnf}), x=1,2 \ldots X$, fission of relevant equilibrated uranium nuclei, is calculated as

$$
\sigma_{\mathrm{nF}}\left(E_{n}\right)=\sigma_{\mathrm{nf}}\left(E_{n}\right)+\sum_{x=1}^{X} \sigma_{\mathrm{n}, \mathrm{xnf}}\left(E_{n}\right)
$$

emissive fission contributions are calculated using $P_{f x}^{J \pi}$ fission probability estimates of ${ }^{239-x} \mathrm{U}$ nuclei:

$$
\sigma_{\mathrm{n}, \mathrm{xnf}}\left(E_{n}\right)=\sum_{J \pi}^{J} \int_{0}^{U_{\max }} W_{x+1}^{J \pi}(U) P_{f(x+1)}^{J \pi}(U) d U
$$

where $W_{x+1}^{J \pi}$ is the population of $(x+1)$-th nucleus at excitation energy $U$, formed after emission of $x$ neutrons, excitation energy $U_{\max }$ is defined by the incident neutron energy $E_{n}$ and energy, removed from the composite system by ${ }^{238} \mathrm{U}(\mathrm{n}, \mathrm{xnf})$ reaction neutrons. Fission probability $P_{f x}^{J \pi}$ of ${ }^{238} \mathrm{U}$ could be almost unambiguously defined by the consistent description of ${ }^{238} \mathrm{U}(\mathrm{n}, \mathrm{F}),{ }^{238} \mathrm{U}(\mathrm{n}, 2 \mathrm{n})$ and prompt fission neutron spectra (PFNS) of ${ }^{238} \mathrm{U}(\mathrm{n}, \mathrm{F})$ in the emissive fission domain $[6,10]$. The exclusive prefission neutron spectrum of ${ }^{238} \mathrm{U}(\mathrm{n}, \mathrm{nf})$ reaction is much sensitive to the fission probability of ${ }^{238} \mathrm{U}$. Consistent description of ${ }^{238} \mathrm{U}(\mathrm{n}, \mathrm{F})$ cross section and PFNS data [11] was confirmed recently, when precise PFNS data by Lovchikova et al. [12] at $\mathrm{E}_{n} \sim 6$ and $7 \mathrm{MeV}$ at were released (see Figs. 1, 2). The previous attempts [13-16] to fill-in the part of the PFNS with $\varepsilon \leq 1.5 \mathrm{MeV}$ by increasing the contribution of the prompt neutrons, coming from the fission fragments of the cooled-down nuclide ${ }^{238} \mathrm{U}$, failed to reproduce the shape of the PFNS. The predicted in $[2,6]{ }^{237} \mathrm{U}(\mathrm{n}, \mathrm{f})$ cross section is compatible with the surrogate data [5], ratio surrogate data by Burke et al. [1] and ratio surrogate data by Burke [7] down to $\sim 0.6 \mathrm{MeV}$ (see Fig. 3). The ratio surrogate data, obtained via ${ }^{238} \mathrm{U}\left(\mathrm{d}, \mathrm{d}^{\prime} \mathrm{F}\right.$ ) and ${ }^{236} \mathrm{U}\left(\mathrm{d}, \mathrm{d}^{\prime} \mathrm{F}\right)$ reactions by Plettner et al. [17], only marginally supported the predicted in $[2]{ }^{237} \mathrm{U}(\mathrm{n}, \mathrm{f})$ cross section. The older neutron-induced fission data [18] McNally et al. and surrogate data by Cramer and Britt [19] overshoot the present estimate and surrogate [5] and ratio surrogate [1] data. In case of data of [18] it is explained in [5] by the ${ }^{237} \mathrm{~Np}$ impurity.

At lower energies the ${ }^{237} \mathrm{U}(\mathrm{n}, \mathrm{f})$ cross section $\left(\mathrm{I}_{\text {g.s. }}=1 / 2\right)$ is defined by $\mathrm{K}^{\pi}=0^{+}$and $\mathrm{K}^{\pi}=1^{+}$ transition state bands at inner (higher) saddle (see [2] and Fig. 3). The position of the abnormal rotational band-head $2^{+}$states at the inner saddle of ${ }^{238} \mathrm{U}$ is influenced by the non-axiality of the 


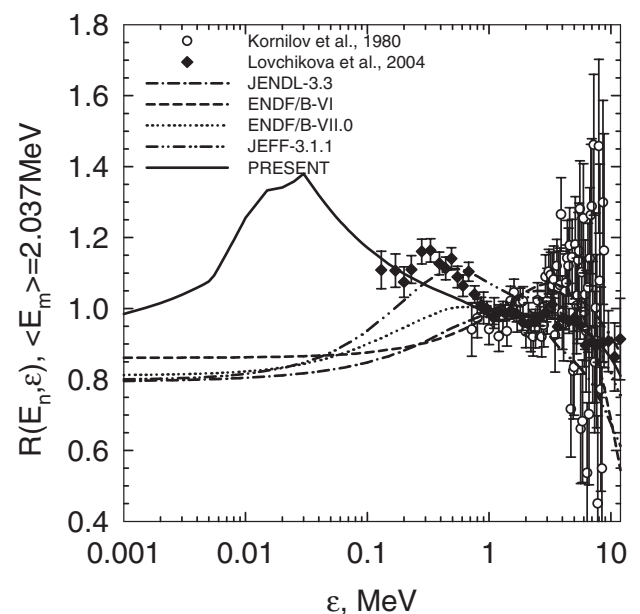

Fig. 1. PFNS of ${ }^{238} \mathrm{U}(\mathrm{n}, \mathrm{F}), E_{n}=6 \mathrm{MeV}$.

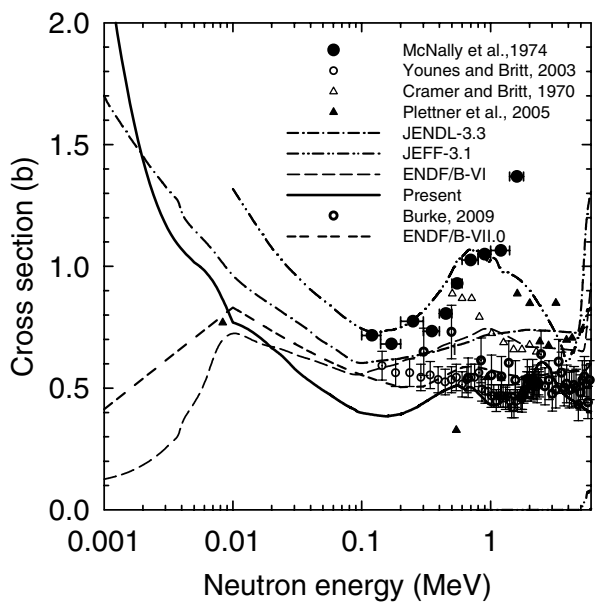

Fig. 3. Fission cross section of ${ }^{237} \mathrm{U}(\mathrm{n}, \mathrm{F})$.

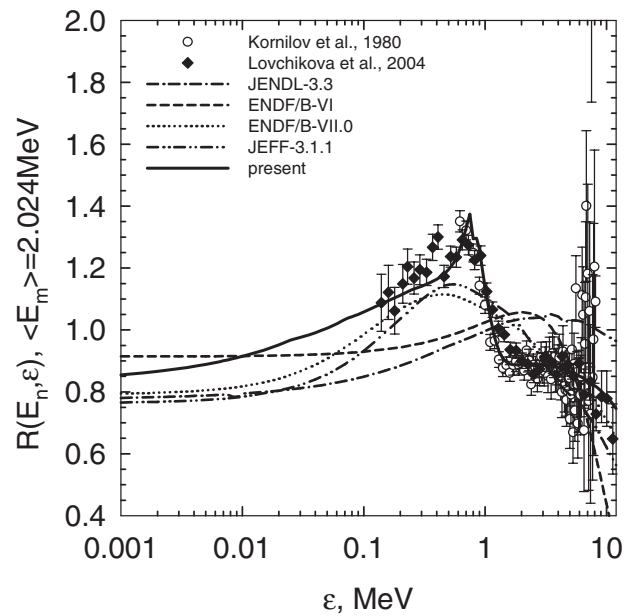

Fig. 2. PFNS of ${ }^{238} \mathrm{U}(\mathrm{n}, \mathrm{F}), E_{n}=7 \mathrm{MeV}$.

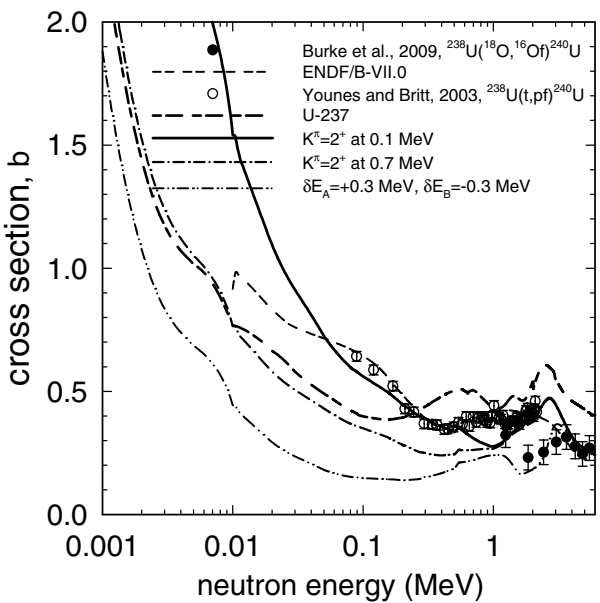

Fig. 4. Fission cross section of ${ }^{239} \mathrm{U}(\mathrm{n}, \mathrm{F})$.

saddle shape. In case of ${ }^{237} \mathrm{U}(\mathrm{n}, \mathrm{f})$ reaction, the excitation/position of the $\mathrm{K}^{\pi}=2^{+}$abnormal rotational band-head at the inner saddle of fissioning nuclide ${ }^{238} \mathrm{U}$ was shown to be marginally important only in the $\mathrm{E}_{n} \sim 0.1-0.8 \mathrm{MeV}$ energy range. For nuclide ${ }^{239} \mathrm{U}$ the position of the $\mathrm{K}^{\pi}=2^{+}$band may strongly influence the ${ }^{239} \mathrm{U}(\mathrm{n}, \mathrm{f})$ cross section shape at $\mathrm{E}_{n} \leq 1 \mathrm{MeV}$ due to higher fission threshold and ground state spin $\mathrm{I}_{\text {g.s. }}=5 / 2$. Fig. 4 demonstrates that the ratio surrogate data, obtained usinf the two-nucleon transfer reactions ${ }^{238} \mathrm{U}\left({ }^{18} \mathrm{O},{ }^{16} \mathrm{OF}\right)$ and ${ }^{234} \mathrm{U}\left({ }^{18} \mathrm{O},{ }^{16} \mathrm{OF}\right)$ by Burke et al. [7], relevant for the ${ }^{239} \mathrm{U}(\mathrm{n}, \mathrm{F})$ and ${ }^{235} \mathrm{U}(\mathrm{n}, \mathrm{F})$ reactions, are discrepant with the stripping reaction ${ }^{238} \mathrm{U}\left({ }^{3} \mathrm{H}\right.$, pf $)$ data $[3,5]$. The sensitivity of the calculated ${ }^{239} \mathrm{U}(\mathrm{n}, \mathrm{F})$ fission cross section to the position of the $\mathrm{K}^{\pi}=2^{+}$band may be an indication that the $\mathrm{J}^{\pi}=2^{+}$states are not excited in the $\left({ }^{18} \mathrm{O},{ }^{16} \mathrm{OF}\right)$ reaction. The fission probability of ${ }^{236} \mathrm{U}$, fissioning in the reaction ${ }^{234} \mathrm{U}\left({ }^{18} \mathrm{O},{ }^{16} \mathrm{OF}\right)$ [7], might not be sensitive to the excitation of $\mathbf{J}^{\pi}=2^{+}$states. Unlike the ${ }^{238} \mathrm{U}$ nuclide, ${ }^{236} \mathrm{U}$ has a higher outer saddle and of critical value is the position of $\mathrm{K}^{\pi}=0^{-}$transition band [20]. That means even in the ratio surrogate data of [7] the effect of missing $\mathbf{J}^{\pi}=2^{+}$would not be cancelled out. That reasoning partly explains the differences of calculated ${ }^{239} \mathrm{U}(\mathrm{n}, \mathrm{f})$ cross section and ratio surrogate data [7] at low incident neutron energies. The ${ }^{238} \mathrm{U}\left({ }^{3} \mathrm{H}\right.$, pf) surrogate data [5] are quite compatible with the calculated ${ }^{239} \mathrm{U}(\mathrm{n}, \mathrm{f})$ cross section (see Fig. 4). 


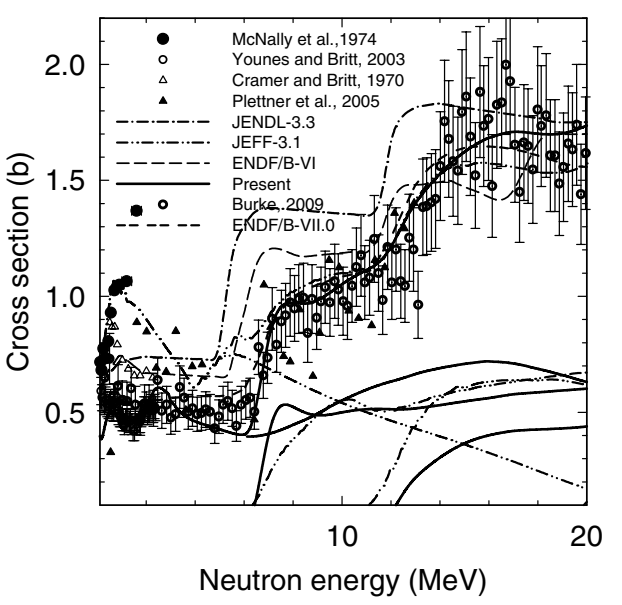

Fig. 5. Fission cross section of ${ }^{237} \mathrm{U}(\mathrm{n}, \mathrm{f})$.

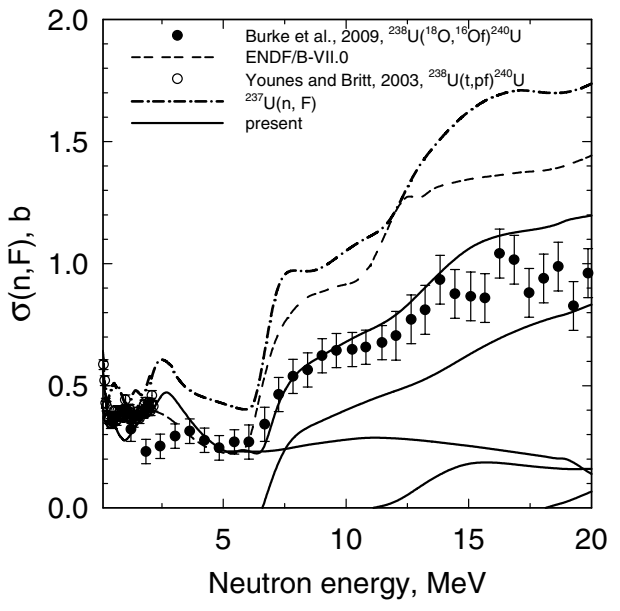

Fig. 6. Fission cross section of ${ }^{239} \mathrm{U}(\mathrm{n}, \mathrm{F})$.

The ratio surrogate data, obtained using ${ }^{238} \mathrm{U}\left(\alpha, \alpha^{\prime} \mathrm{F}\right)$ and ${ }^{236} \mathrm{U}\left(\alpha, \alpha^{\prime} \mathrm{F}\right)$ reactions by Burke [6], which are scattering much less than those of [1], strongly confirm the predicted in [2] ${ }^{237} \mathrm{U}(\mathrm{n}, \mathrm{f})$ cross section from $\mathrm{E}_{n} \sim \mathrm{E}_{n, n f}$ up to $E_{n} \sim 20 \mathrm{MeV}$ (see Fig. 3), here $E_{n, n f}$ is the (n, nf) fission reaction threshold. The contribution of the first-chance fission reaction ${ }^{237} U(n, f)$ resembles the increasing trend of the observed ${ }^{237} \mathrm{U}(\mathrm{n}, \mathrm{f})$ cross section, while the contribution of ${ }^{237} \mathrm{U}(\mathrm{n}, \mathrm{nf})$ reaction remains rather flat. That partitioning of the fission chances contributions to the observed fission cross section is similar to that proposed for the ${ }^{235} \mathrm{U}(\mathrm{n}, \mathrm{F})[21]$. At $\mathrm{E}_{n}<\mathrm{E}_{n, n f}$ the cross section shape is defined mostly by the fission barrier parameters and washing -out of pairing effects in the level density of even ${ }^{238} \mathrm{U}$ nuclide. The increasing trend of the first chance fission cross section ${ }^{237} \mathrm{U}(\mathrm{n}, \mathrm{f})$ above $E_{n, n f}$ is explained by the shell effects in the level densities of fissioning ${ }^{238} \mathrm{U}$ and residual ${ }^{237} \mathrm{U}$ nuclides.

At $\mathrm{E}_{n}$ higher than $\sim 4 \mathrm{MeV}$ the ${ }^{239} \mathrm{U}(\mathrm{n}, \mathrm{F})$ data, obtained using ratio surrogate data of ${ }^{238} \mathrm{U}\left({ }^{18} \mathrm{O},{ }^{16} \mathrm{OF}\right){ }^{240} \mathrm{U}$ and ${ }^{234} \mathrm{U}\left({ }^{18} \mathrm{O},{ }^{16} \mathrm{OF}\right){ }^{2360} \mathrm{U}$ are consistent with the statistical theory approach, proven in case of neutron-induced fission of ${ }^{237} \mathrm{U}$ target nuclide (see Fig. 6). The fission probabilities of ${ }^{239} \mathrm{U}$ and ${ }^{238} \mathrm{U}$, fissioning in ${ }^{239} \mathrm{U}(\mathrm{n}, \mathrm{nf})$ and ${ }^{239} \mathrm{U}(\mathrm{n}, 2 \mathrm{nf})$ are fixed using ${ }^{238} \mathrm{U}(\mathrm{n}, \mathrm{F})$ and ${ }^{237} \mathrm{U}(\mathrm{n}, \mathrm{f})$ reaction cross sections. The increasing trend of the observed ${ }^{239} \mathrm{U}(\mathrm{n}, \mathrm{F})$ reaction cross section is explained by the ${ }^{239} \mathrm{U}(\mathrm{n}, \mathrm{nf})$ reaction contribution. The evaluation of ENDF/B-VII.0 [14] greatly overestimates the surrogate measured ${ }^{239} \mathrm{U}(\mathrm{n}, \mathrm{F})$ cross section. The contributions of ${ }^{239} \mathrm{U}(\mathrm{n}, \mathrm{f}),{ }^{239} \mathrm{U}(\mathrm{n}, \mathrm{nf})$ and ${ }^{239} \mathrm{U}(\mathrm{n}, 2 \mathrm{nf})$ reactions of ENDF/B-VII.0 are unavailable, but it might be concluded, that whatever they are, they are inconsistent with ours. The calculated ${ }^{239} \mathrm{U}(\mathrm{n}, \mathrm{F})$ cross section of [2], is not only systematically lower than that of ${ }^{237} \mathrm{U}(\mathrm{n}, \mathrm{f})$, but has different fission chances contributions.

\section{$3^{230} \mathrm{Th}(\mathrm{n}, \mathrm{F})$ and ${ }^{231} \mathrm{Th}(\mathrm{n}, \mathrm{F})$ reactions}

It was shown in [9], that the ${ }^{230} \mathrm{Th}(\mathrm{n}, \mathrm{F})$ cross section cross section shape in the emissive fission domain is quite similar to that of ${ }^{232} \mathrm{Th}(\mathrm{n}, \mathrm{F})$. The surrogate data for ${ }^{230} \mathrm{Th}(\mathrm{n}, \mathrm{F})$ reaction cross section, inferred from the ratio of fission probabilities of ${ }^{231} \mathrm{Th}$ and ${ }^{235} \mathrm{U}$ nuclides by Goldblum et al. [8], confirm that finding, which was initially based on data by Meadows [22,23] (see Fig. 7). The desired compound nuclides were populated using a pick-up reactions ${ }^{232} \mathrm{Th}\left({ }^{3} \mathrm{He},{ }^{4} \mathrm{He}\right)$ and ${ }^{236} \mathrm{U}\left({ }^{3} \mathrm{He},{ }^{4} \mathrm{He}\right)$. At medium excitations of $10-15 \mathrm{MeV}$, when first pre-fission neutron emission is possible, the secondchance fission probability of ${ }^{231} \mathrm{Th}$ is defined by the excitation of $\mathrm{K}^{\pi}=0^{-}$states, which depends on the mass-asymmetry of the ${ }^{230} \mathrm{Th}$ nuclide saddle shapes. The shape of the surrogate data around $\mathrm{E}_{n} \sim 10 \mathrm{MeV}$ resembles that of the calculated cross section, specifically, the step-like shape is attributed to the excitation of the two-quasi-particle states in ${ }^{230} \mathrm{Th}$ fissioning nuclide. At $\mathrm{E}_{n}<10 \mathrm{MeV}$ the ${ }^{230} \mathrm{Th}(\mathrm{n}, \mathrm{F})$ cross section is defined by the collective levels, lying within the pairing gap of ${ }^{230} \mathrm{Th}$. 


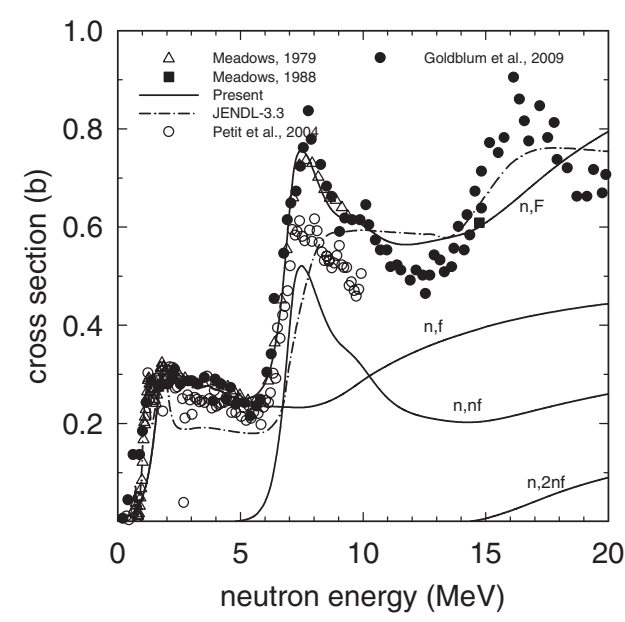

Fig. 7. Fission cross section of ${ }^{230} \mathrm{Th}(\mathrm{n}, \mathrm{F})$.

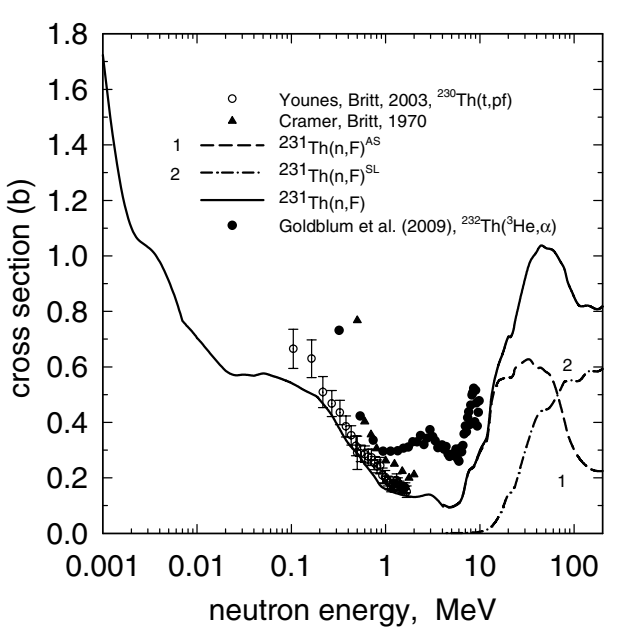

Fig. 8. Fission cross section of ${ }^{231} \mathrm{Th}(\mathrm{n}, \mathrm{F})$.

The surrogate data ${ }^{232} \mathrm{Th}\left({ }^{3} \mathrm{He},{ }^{4} \mathrm{He}\right){ }^{231} \mathrm{Th}$ [24], corresponding to incident neutron energies higher than the $\mathrm{E}_{n, n f}$ are systematically lower than both neutron-induced [22,23] and ratio surrogate data [8]. That means in the emissive fission domain the fission cross section can not be obtained multiplying the measured fission probability by the neutron absorption cross section. Similar conclusion was reached for the ${ }^{231,232,233} \mathrm{~Pa}(\mathrm{n}, \mathrm{F})$ cross sections $[25,26]$ That is a failure of the "factorization theorem".

The surrogate data for the ${ }^{231} \mathrm{Th}(\mathrm{n}, \mathrm{F})$ reaction are inferred from the ratio of fission probabilities of ${ }^{232} \mathrm{Th}$ and ${ }^{236} \mathrm{U}$ nuclides by Goldblum et al. [8]. The desired compound nuclei were populated using inelastic scattering reactions ${ }^{232} \mathrm{Th}\left({ }^{3} \mathrm{He},{ }^{3} \mathrm{He}^{\prime}\right)$ and ${ }^{236} \mathrm{U}\left({ }^{3} \mathrm{He},{ }^{3} \mathrm{He}^{\prime}\right)$ by Goldblum et al. [8]. Even nuclide ${ }^{232} \mathrm{Th}$ mass-asymmetric saddle shape is probed in the first chance fission reaction. For the neutron-induced fission cross section ${ }^{231} \mathrm{Th}(\mathrm{n}, \mathrm{f})$ the $\mathrm{K}^{\pi}=0^{-}$states, lying within the pairing gap, contribute via the p-wave neutrons, they define fission cross section shape at tens $50-700 \mathrm{keV}$ energy range. The calculated ${ }^{231} \mathrm{Th}(\mathrm{n}, \mathrm{f})$ reaction cross section is obtained by extracting ${ }^{232} \mathrm{Th}$ relevant fission and ${ }^{232,231} \mathrm{Th}$ level density parameters via ${ }^{232} \mathrm{Th}(\mathrm{n}, \mathrm{F})$ data analysis, as it was done for the ${ }^{237} \mathrm{U}(\mathrm{n}, \mathrm{f})$ reaction. The step-like shape of the cross section around $\mathrm{E}_{n} \sim 100 \mathrm{keV}$ is defined by the lowered position of the $\mathrm{K}^{\pi}=0^{-}$states, it is supported by surrogate data of ${ }^{230} \mathrm{Th}(\mathrm{t}, \mathrm{pf})$ reaction [5]. The ratio surrogate data of [8] are in severe conflict with surrogate and calculated data on ${ }^{231} \mathrm{Th}(\mathrm{n}, \mathrm{f})$ reaction cross section, being systematically higher (see Fig. 8). That contradiction can not be explained by the sensitivity of the spin distribution of the excited states in ${ }^{232} \mathrm{Th}$ and ${ }^{236} \mathrm{U}$ nuclides, as it was in case of ${ }^{239} \mathrm{U}(\mathrm{n}, \mathrm{F})$ reaction.

\section{Conclusions}

Analysis of the consistency of surrogate, ratio surrogate and neutron-induced data itself for a chain of Th and U nuclides evolved a number of restrictions. The surrogate data hardly could be used for the excitation energies, corresponding to emissive fission domain. Theoretical estimate of ${ }^{237} \mathrm{U}(\mathrm{n}, \mathrm{f})$, based on analysis of the second chance fission effects in observed fission cross section and prompt fission neutron spectra of ${ }^{238} \mathrm{U}(\mathrm{n}, \mathrm{F})$ is strongly confirmed by ratio surrogate data. Similar approach, pinned down at $\mathrm{E}_{n} \leq 2 \mathrm{MeV}$ to surrogate data, allows to reproduce newly reported ratio surrogate data for the ${ }^{239} \mathrm{U}(\mathrm{n}, \mathrm{F})$. Restrictions for the usage of ratio surrogate data are revealed. The structure of the fission barrier and the angular moments of the entrance channel states are shown to be significant for the ratio surrogate measurements. That means the effect of missing certain important $\mathbf{J}^{\pi}$ states might not be cancelled out as dependent on the neutron target nuclide ground state spin and fission barrier structure. The excitation/position of the $\mathrm{K}^{\pi}=2^{+}$abnormal rotational band-head at the inner saddle of even-even 
$\mathrm{U}$ fissioning nuclide like ${ }^{240} \mathrm{U}$ is shown to be critically important. At ${ }^{231} \mathrm{Th}$ nuclide medium excitations of $10-20 \mathrm{MeV}$, when pre-fission neutron emission is possible in the reaction ${ }^{232} \mathrm{Th}\left({ }^{3} \mathrm{He},{ }^{4} \mathrm{He}\right){ }^{231} \mathrm{Th}$, the excitation of $\mathrm{K}^{\pi}=0^{-}$states is "amplified" by the mass-asymmetry of the even nuclide ${ }^{230} \mathrm{Th}$ saddle shapes. Octupole band states are shown to be relevant also for the ${ }^{232} \mathrm{Th}\left({ }^{3} \mathrm{He},{ }^{3} \mathrm{He}^{\prime}\right)^{232} P$ Th reaction. Surrogate reaction data based on inelastic scattering of ${ }^{3} \mathrm{He}$, can not be used for the ${ }^{231} \mathrm{Th}(\mathrm{n}, \mathrm{F})$ fission cross section estimation.

\section{References}

1. J.T. Burke, L.A. Bernstein, J. Escher et al., Phys. Rev. C 73, 054604 (2006)

2. V.M. Maslov, Phys. Rev. C 72, 044607 (2005)

3. H.C. Britt, J.B. Wilhelmy, Nucl. Sci. Eng. 72, 222 (1979)

4. W. Younes and H.C. Britt, Phys. Rev. C 67, 024610 (2003)

5. W. Younes and H.C. Britt, Phys. Rev. C 68, 034610 (2003)

6. V.M. Maslov et al. Phys. Rev. C69, 034607 (2004)

7. J.T. Burke, $4^{\text {th }}$ International Workshop on Fission, 13-16 May, 2009, CEA, Cadarache, France.

8. B.L. Goldblum, S.R. Stroberg, J.L. Allmond et al. Phys. Rev. C80, 044610 (2009)

9. V.M. Maslov, Nucl. Phys. A 743, 236 (2004)

10. V.M. Maslov, Phys. Atom. Nucl. 71, 9 (2008)

11. N.V. Kornilov, B.V. Zhuravlev, O.A. Salnikov et al. Proc. of the 5-th Conf. on Neutron Physics, Kiev, September, 15-19,1980, vol. 2, p. 44, 1980.

12. G.N. Lovchikova, A.M. Trufanov, and M.I. Svirin, Phys. At. Nucl. 67, 1246 (2004)

13. R.W. Roussin, P.G. Young, R. McKnight Proc. Int. Conf. Nucl. Data for Sci. and Tech., Gatlinburg, USA, May 9-13, 1994, J.K. Dickens (Ed.), ANS (1994) p. 692.

14. M.B. Chadwick, P. Oblozinsky, M. Herman et al. Nucl. Data Sheets, 107, 2931 (2006)

15. JEFF 3.1.1. Nuclear Data Library Report 21, NEA No 6190. OECD, 2006

16. K. Shibata et al. Journ. Nucl. Sci. Technol. 39, 1125 (2002)

17. C. Plettner, H. Ai, C.W. Beausange et al. Phys. Rev. C71, 051602(R) (2005)

18. J.H. McNally, J.W. Barnes, B.J. Dropesky et al., Phys. Rev. C9, 717 (1974)

19. J.D. Cramer and H.C. Britt, Nucl. Sci. Eng., 41,177 (1970)

20. V.M. Maslov, Nuclear Data for Science and Technology (O. Bersillon, F. Gunsing,

E. Bauge, R. Jacqmin and S. Leray, Eds.), Nice, France, April 22-27, 2007, EDP Sciences, p. 475.

21. V.M. Maslov, N.V. Kornilov, A. B. Kagalenko et al. Nucl. Phys. A760, 274 (2005)

22. J.W. Meadows, Proc. Int. Conf. on Nuclear Cross Sections for Technology, Knoxville, Tennessee, 22-26 Oct 1979, p. 479

23. J.W. Meadows, Journ. Ann. Nucl. Energy, 15, 421 (1988).

24. M. Petit, M. Aiche, G. Barreau et al. Nucl. Phys. A, 735, 3 (2004)

25. V.M. Maslov, Eur. Phys. Journ., EPJ Web of Conferences Proceedings, CNR09, Second International Workshop on Compound Nuclear Reactions and Related Topics, p. 12001, 2010.

26. V.M. Maslov, EFNUDAT Fast Neutrons, Proc. of the Scientific Workshop on Neutron Measurements, Theory and Applications, 28-30 April, 2009, Geel, Belgium, p. 105. 